\title{
Ginsenoside RbI Protects Against Diabetic Cardiomyopathy by Regulating the Adipocytokine Pathway
}

\author{
Chenyang Zhang' \\ Meixin $\mathrm{Han}^{2}$ \\ Xuelian Zhang' \\ Hongna Tong' \\ Xiaobo Sun' \\ Guibo Sun' \\ 'Institute of Medicinal Plant \\ Development, Peking Union Medical \\ College and Chinese Academy of Medical \\ Sciences, Beijing, People's Republic of \\ China; ${ }^{2}$ College of Pharmacy, Harbin \\ University of Commerce, Harbin, \\ People's Republic of China
}

Correspondence: Xiaobo Sun; Guibo Sun Emailsun_xiaobol63@I63.com; sunguibo@।26.com
Purpose: Obesity and diabetes are often accompanied by chronic inflammation and insulin resistance, which lead to complications such as diabetic cardiomyopathy. Ginsenoside Rb1 has been used to treat diabetes and obesity and reduce inflammation as well as risk of heart diseases. However, the role of ginsenoside Rb1 in treating diabetic cardiomyopathy remains unclear.

Methods: Diabetic mice were administered ginsenoside Rb1 for 12 weeks, and their body weight, body fat, and blood glucose levels as well as and serum insulin, lipids, and adipocytokine levels were assessed. Lipid accumulation, pathological morphology of the adipose tissue, liver, and heart were examined. Western blot and qRT-PCR were performed to investigate the molecular changes in response to ginsenoside $\mathrm{Rb} 1$ treatment.

Results: Ginsenoside Rb1 treatment significantly reduced body weight and body fat, attenuated hyperglycemia and hyperlipidemia, and ameliorated insulin resistance and abnormal levels of adipocytokines in diabetic mice. In addition, lipid accumulation and inflammation reduced while the functions of heart improved in the ginsenoside Rb1-treated group. Furthermore, antioxidant function improved in the ginsenoside Rb1-treated diabetic hearts. PCR and Western blotting analyses revealed that the lipid-lowering effect of ginsenoside Rb1 and the resulting improvement of cardiac function could be attributed to the adipocytokine pathway, which promoted energy homeostasis and alleviated cardiac dysfunction.

Conclusion: Ginsenoside Rb1 lowered lipid levels in a adipocytokine-mediated manner and attenuated hyperglycemia/hyperlipidemia-induced oxidative stress, hypertrophy, inflammation, fibrosis, and apoptosis in cardiomyocytes.

Keywords: diabetic cardiomyopathy, ginsenoside Rb1, adipocytokines, lipid metabolism, oxidative stress, inflammation

\section{Introduction}

Type 2 diabetes mellitus is associated with obesity and insulin resistance, ${ }^{1}$ with insulin and leptin resistance being central pathophysiological features of obesity. ${ }^{2}$ An excess of adipose tissue, as seen in obesity, leads to abnormal blood levels of free fatty acids and adipocytokines that affect normal tissue function. ${ }^{3,4}$ Adipose tissue plays a crucial role in regulating systemic lipid metabolism and may influence cardiovascular health. ${ }^{5}$ Diabetic cardiomyopathy is a complication of diabetes and can lead to heart failure, a cause of high mortality and poor prognosis in patients with diabetes. ${ }^{6}$ Diabetes cardiomyopathy is characterized by high lipid intake and accumulation, as well as low in glucose utilization by cardiomyocytes 
that induces myocardial reactive oxygen species (ROS) accumulation, inflammation, and fibrosis, and leads to myocardial apoptosis and heart failure. ${ }^{7,8}$ Although several studies have focused on diabetic cardiomyopathy, few have addressed the role of adipose tissue in this disease, and none of the drugs tested in animals has so far been used clinically.

Adipocytokines are secreted from adipose tissue, and play critical roles in energy homeostasis. ${ }^{9}$ In diabetes and obesity, abnormal release of adipocytokines into the circulating blood affects metabolism in various tissues and organs, may influence insulin sensitivity, and induce systemic complications. ${ }^{9}$ Obesity-associated abnormal secretion of adipocytokines is an important biomarker of poor cardiovascular outcomes, suggesting that adipocytokines play a crucial role in obesity-associated cardiovascular disorders. ${ }^{10}$ However, the comprehensive evaluation of role played by adipocytokines in diabetic cardiomyopathy is pending. Therefore, it is important to map the downstream signaling pathways of adipocytokines involved in metabolic disorders to identify new therapeutic targets and drugs for metabolic diseases and their associated complications. ${ }^{11}$

The majority of drugs evaluated for the treatment of diabetes have focused on specific targets; however, ginsenoside Rb1, a major saponin from Panax notoginseng, has been used to treat diabetes and obesity with multi-target effects. ${ }^{12}$ Ginsenoside Rb1 promotes browning of 3T3-L1 preadipocytes and thermogenesis in diabetic mice. ${ }^{13} \mathrm{In}$ high-fat diet (HFD)-fed obese mice, ginsenoside Rb1 alleviated insulin resistance and reduced lipid accumulation in liver. ${ }^{14}$ Moreover, ginsenoside Rb1 alleviated ischemia/ reperfusion injury and heart failure. ${ }^{15}$ Although ginsenoside $\mathrm{Rb} 1$ reportedly improves symptoms of cardiomyopathy by regulating calcium signaling, ${ }^{16}$ the precise mechanism by which ginsenoside $\mathrm{Rb} 1$ exerts protective effects in diabetes, obesity, and complications such as diabetic cardiomyopathy remain unknown.

The goal of this study was to investigate the effect and mechanism of ginsenoside $\mathrm{Rb} 1$ in diabetic cardiomyopathy and confirm the roles of adipocytokines in diabetes and diabetic cardiomyopathy. We observed that ginsenoside $\mathrm{Rb} 1$ treatment reduced body weight and serum lipid levels; improved lipid accumulation, inflammation, and function in the heart. Moreover, ginsenoside Rb1 regulated the secretion of adipocytokines and expression of genes and proteins related to glucose and lipid metabolism. These findings suggest that ginsenoside $\mathrm{Rb} 1$ may protect against heart failure in diabetes via modulating the adipocytokine pathway, which might be a potential therapy in treating diabetic cardiomyopathy.

\section{Materials and Methods}

\section{Animal Experiments}

All animal experiments were conducted in accordance with the guidelines of National Institutes of Health Guide for the Care and Use of Laboratory Animals and approved by the Laboratory Animal Ethics Committee of the Institute of Medicinal Plant Development, Peking Union Medical College. Eight-week-old male $\mathrm{db} / \mathrm{m}$ and $\mathrm{db} / \mathrm{db}$ mice were purchased from Vital River Laboratory Animal Technology Co., Ltd. (Beijing, China). All animals were housed in a room with a temperature at $22{ }^{\circ} \mathrm{C}$ and a $12 \mathrm{~h}$ light-dark cycle. The animals had free access to food and water until 36 weeks of age. Animals were randomly sorted into six groups of 10 mice each: (1) db/m (Control), (2) db/db (Model), (3) db/db + metformin $250 \mathrm{mg} / \mathrm{kg} /$ day (metformin), (4) db/db + ginsenoside Rb1 $100 \mathrm{mg} / \mathrm{kg} /$ day (H-Rb1), (5) db/db + ginsenoside Rb1 $50 \mathrm{mg} / \mathrm{kg} /$ day (M$\mathrm{Rb} 1$ ), and (6) db/db + ginsenoside Rb1 $25 \mathrm{mg} / \mathrm{kg} /$ day (L$\mathrm{Rb} 1)$. Ginsenoside Rb1 or metformin was administered by gavage from week 24 every day for 12 weeks. Ginsenoside $\mathrm{Rb} 1$ was purchased from Chengdu Must Bio-technology Co., Ltd. (Chengdu, China), and metformin was obtained from Sino American Shanghai Squibb Pharmaceutical Co., Ltd. (Shanghai, China). Mice in the control and model groups were administered pure water.

\section{Blood Tests}

After 12 weeks of treatment, mice were fasted overnight, and fasting blood glucose levels were measured using a blood glucose meter. Blood samples were extracted from the retro-orbital area and centrifuged at $3000 \times \mathrm{g}$ for 15 min to obtain serum samples. Lipid contents, liver function markers, and serum proteins were analyzed using a fully automatic biochemical analyzer (AU480, Beckman Coulter, Pasadena, CA, USA). Lipid content measurements included total triglyceride (TG), total cholesterol (CHO), high-density lipoprotein (HDL), and low-density lipoprotein (LDL). Measured liver function markers included alanine aminotransferase (ALT) and aspartate aminotransferase (AST). Serum measurements included creatine kinase (CK), creatine kinase-MB (CK-MB), and lactate dehydrogenase (LDH). Assay kits for catalase (CAT), malondialdehyde (MDA), superoxide dismutase 
(SOD), and glutathione peroxidase (GSH-px) (Nanjing Jiancheng Bioengineering Institute, China) were used to detect serum lipid peroxidation, according to manufacturer instructions. Levels of insulin, free fatty acid (FFA), adiponectin, and leptin, as well proinflammatory cytokines such as interleukin- 6 (IL-6), interleukin $1 \alpha$ (IL-1 $\alpha$ ), monocyte chemoattractant protein-1 (MCP-1), tumor necrosis factor- $\alpha$ (TNF- $\alpha$ ), and C-reactive protein (CRP) were quantified using respective kits according to manufacturer instructions (Beijing Huaying Institute of Biological Technology, China).

\section{Echocardiography}

After 12 weeks of treatment, echocardiography was performed using the Vevo 770 Imaging System (VisualSonics, Canada), and each parameter was measured twice. All mice were anesthetized with isoflurane (RDW, China). Echocardiographic parameters were evaluated in the parasternal long-axis view. The left ventricular internal diameter (LVID), left ventricular anterior wall thickness (LVAW), and left ventricular posterior wall thickness (LVPW) were measured. Left ventricular ejection fraction (EF) and fractional shortening (FS) were calculated.

\section{Histology}

Mouse hearts were fixed in formalin and embedded in paraffin for histological analysis. Samples were cut into 5 $\mu \mathrm{m}$ thick sections and subjected to hematoxylin and eosin (HE), Masson's trichrome, and Oil Red O stainings and TdT-mediated dUTP nick-end labeling (TUNEL). Images were acquired using a panoramic scanning microscope (3DHISTECH, Hungary) and processed with Caseviewer version 2.4 (3DHISTECH). The livers of mice were also stained with HE and Oil Red $\mathrm{O}$ after fixation and embedding and evaluated.

\section{Cell Culture and Treatment}

Rat embryonic cardiac myoblasts (H9c2) were purchased from the Cell Bank of the Chinese Academy of Sciences (Shanghai, China). Dulbecco's Modified Eagle's Medium (DMEM) supplemented with $10 \%$ fetal bovine serum (FBS) was used to culture cells at $37^{\circ} \mathrm{C}$ under a $5 \% \mathrm{CO}_{2}$ atmosphere. When the cells reached approximately $80 \%$ confluence, they were pre-treated with different concentrations of ginsenoside $\mathrm{Rb} 1$ or $5 \mu \mathrm{M}$ berberine for $24 \mathrm{~h}$, and then incubated in FBS-free DMEM with or without palmitate acid (PA) for $24 \mathrm{~h}$. Mouse preadipocytes, 3T3-L1 cells, were purchased and maintained in the same conditions as those of the H9c2 cells. Two days after the cells reached confluence, differentiation was induced by culturing them for $48 \mathrm{~h}$ in presence of $1 \mu \mathrm{M}$ dexamethasone, $0.5 \mathrm{mM} 3$-isobutyl-1-methylxanthine, and $10 \mu \mathrm{g} / \mathrm{mL}$ insulin added to DMEM with $10 \%$ FBS. Two days later, the medium was replaced with a differentiation medium $(10 \mu \mathrm{g} / \mathrm{mL}$ insulin in DMEM with $10 \%$ FBS). After $48 \mathrm{~h}$ of treatment, the medium was discarded, and the cells were used in subsequent experiments.

\section{Cell Viability}

Cell viability was assessed using 3-(4,5-dimethylthiazol-2yl)-2,5-diphenyltetrazolium bromide (MTT) (Solarbio, China). After seeding in 96-well plates and reaching approximately $80 \%$ confluence, the cells were treated with different concentrations of ginsenoside $\mathrm{Rb} 1$ or $\mathrm{PA}$, and then incubated with $1 \mathrm{mg} / \mathrm{mL}$ MTT for $4 \mathrm{~h}$ at $37^{\circ} \mathrm{C}$ in the dark. Subsequently, the medium was aspirated, and dimethyl sulfoxide was added to dissolve the formazan crystals. The absorbance was measured using a microplate reader at $570 \mathrm{~nm}$.

\section{BODIPY Staining}

BODIPY $^{\mathrm{TM}} 493 / 503$ was used to measure intracellular lipid accumulation in H9c2 and 3T3-L1 cells (Invitrogen, USA). After fixing with $4 \%$ paraformaldehyde, the cells were washed three times and incubated with BODIPY working solution at room temperature in the dark for 30 min. After incubation, the cells were washed three times with phosphate-buffered saline (PBS) for subsequent fluorescence microscopy imaging (Olympus, Japan).

\section{Superoxide Measurement}

Mitochondrial ROS levels were measured using MitoSOX ${ }^{\mathrm{TM}}$ Red (Invitrogen). After washing three times with PBS, H9c2 cells were incubated in dark with MitoSOX ${ }^{\mathrm{TM}}$ Red working solution at $37^{\circ} \mathrm{C}$ for $20 \mathrm{~min}$. The cells were then washed three times with PBS before imaging using a fluorescence microscope (Olympus).

\section{Quantitative Reverse-Transcription PCR (qRT-PCR)}

The quality and quantity of total mRNA isolated from mouse hearts using TRIzol were assessed using the Nanodrop 2000 (Thermo Fisher Scientific, USA). Samples with $260 / 280$ and 260/230 ratios between 1.8 and 2.0 were reverse-transcribed into cDNA and used for 
subsequent experiment. TB Green II (Takara, Japan) was used to quantify the PCR-amplified products, and expression levels were calculated using the $\Delta \Delta \mathrm{Ct}$ method.

\section{Western Blot Analysis}

Samples were incubated with lysis buffer and centrifuged at $4^{\circ} \mathrm{C}$ for $30 \mathrm{~min}$ (CWBIO, China). The supernatants were collected and mixed with loading buffer, and boiled to denature proteins. Protein samples from each group were then loaded into different wells and resolved on an $8 \%$ sodium dodecyl sulfate-polyacrylamide gel via electrophoresis. Proteins were transferred onto nitrocellulose membranes and blocked for $2 \mathrm{~h}$, then incubated with primary antibodies against PGC-1 $\alpha$, PPAR $\alpha$, CPT1A, CPT1B, CPT2, and ACTIN at $4^{\circ} \mathrm{C}$ overnight (Abclonal, China). Membranes were washed three times with TBST and incubated with secondary antibody for $90 \mathrm{~min}$. Next, the protein bands were detected with ECL Western blot detection kits (CWBIO) according to manufacturer instructions, and quantified using ImageJ. All experiments were performed at least in triplicate, and the average values were used for comparisons.

\section{Statistical Analysis}

All statistical analyses were performed using SPSS version 17.0 (SPSS Inc., Chicago, USA). Data are presented as the mean \pm SEM. One-way analysis of variance and least significant difference (LSD) analyses were used to compare multiple groups. Statistical significance was set at $\mathrm{p}<0.05$.

\section{Results}

\section{Ginsenoside RbI Reduced Insulin}

Resistance and Serum Lipid Levels in $\mathrm{db} /$ db Mice

As shown in Figure 1A and B, at 36 weeks, $\mathrm{db} / \mathrm{db}$ mice showed a significant increase in body weight and blood glucose levels compared to the control $(p<0.01$ ). Treatment with metformin, a classical drug used to control blood glucose levels in diabetes, resulted in a significant decrease in body weight and blood glucose levels ( $p<$ $0.01)$. H-Rb1 treatment also reduced body weight and blood glucose of diabetic mice $(p<0.05)$, while M-Rb1 and L-Rb1 treatments only reduced body weight $(\mathrm{p}<$ 0.05). Body fat, serum insulin, and free fatty acid levels were also elevated (Figure $1 \mathrm{C}-\mathrm{E}, \mathrm{p}<0.001$ ), but reduced in the H-Rb1 group $(p<0.05) . M-R b 1$ treatment reduced body fat and serum insulin levels $(\mathrm{p}<0.05)$, whereas L-Rb1 only decreased serum insulin levels $(p<0.05)$. Elevated serum lipid levels were observed in the model group compared to the control $(\mathrm{p}<0.01)$, and treatment with metformin reduced both $\mathrm{CHO}$ and HDL levels $(\mathrm{p}<$ 0.05). Furthermore, $\mathrm{H}-\mathrm{Rb} 1$ treatment improved $\mathrm{CHO}$ and LDL levels in the diabetic mice (Figure 1F-I, p $<0.05$ ). Similarly, M-Rb1 and L-Rb1 groups exhibited decreased CHO and LDL levels compared to the model group; however, the difference was not significant $(\mathrm{p}>0.05)$. Additionally, metformin supplementation decreased serum TG and LDL levels of diabetic mice $(p>0.05)$. Together, these results show that ginsenoside $\mathrm{Rb} 1$

C

D
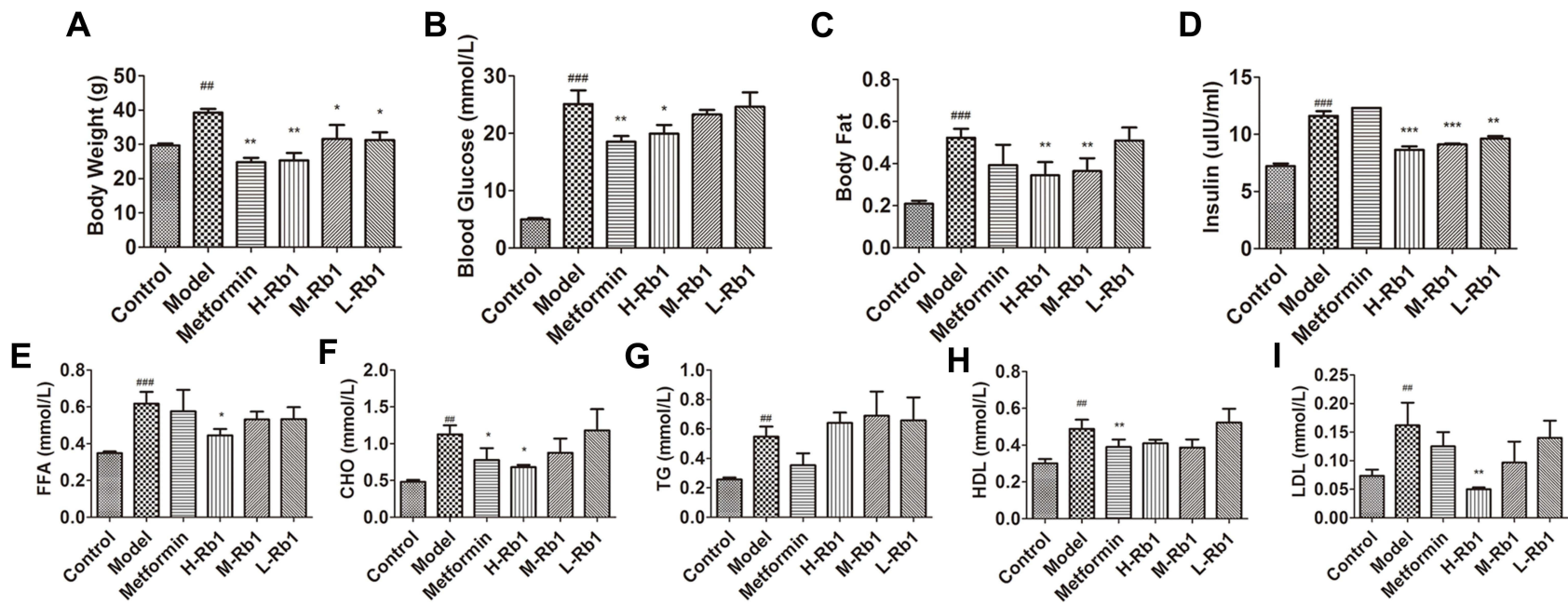

Figure I Ginsenoside RbI reduced lipid accumulation in diabetic mice. (A) body weight; (B) blood glucose level and (C) body fat level of 36-week-old mice after I2 weeks of treatment. (D) serum insulin; (E) FFA; (F) CHO; (G) TG; (H) HDL and (I) LDL in indicated groups. Data are expressed as the mean \pm SEM (n = 3-6). * $<<0.05$ or ${ }^{* *} \mathrm{p}<0.0$ I vs model group; ${ }^{\#} \mathrm{p}<0.01$ or ${ }^{\# \#} \mathrm{p}<0.00$ I vs the control. 
alleviated obesity, hyperglycemia, and hyperlipidemia in diabetic mice.

\section{Ginsenoside RbI Improved Secretion of Adipocytokines and Lipid Accumulation in Adipocytes}

Treatment with ginsenoside $\mathrm{Rb} 1$ significantly reduced inflammation, as evidenced by decreased serum CRP, MCP1, TNF- $\alpha$, IL-1 $\beta$, and IL-6 levels in treated $\mathrm{db} / \mathrm{db}$ mice (Figure $2 \mathrm{~A}-\mathrm{E}, \mathrm{p}<0.01$ ). No reduction in the MCP1 levels was observed $(p>0.05)$ in the L-RB1 group, whereas treatment with metformin reduced MCP1 levels $(\mathrm{p}<0.01)$. Adiponectin levels in the model mice were significantly decreased compared to the control, and $\mathrm{H}-\mathrm{Rb} 1$ and $\mathrm{M}-\mathrm{Rb} 1$ treatments improved adiponectin levels significantly (Figure $2 \mathrm{~F}, \mathrm{p}<0.05$ ). In contrast to the metformin treatment, ginsenoside $\mathrm{Rb} 1$ treatment decreased the elevated leptin levels in diabetic mice (Figure 2G, p < 0.05 ), indicating reduced leptin resistance. Additionally, the 3T3-L1 preadipocytes were incubated with berberine and ginsenoside $\mathrm{Rb} 1$ to detect lipid accumulation. Berberine was used as a positive control as it significantly improves lipid metabolism and reduces lipid levels. ${ }^{17}$ The MTT assay was performed to ensure that the ginsenoside $\mathrm{Rb} 1$ concentration used in the experiments was non-toxic (Figure 2H). Subsequently, $20 \mu \mathrm{M}, 40 \mu \mathrm{M}$, and $80 \mu \mathrm{M}$ ginsenoside $\mathrm{Rb} 1$ were used for further experiments. BODIPY staining on day 4 of differentiation showed a significant decrease in lipid accumulation in berberineand ginsenoside Rb1-treated mature adipocytes (Figure 2I and $\mathrm{J}, \mathrm{p}<0.001$ ). This finding confirmed that both berberine and ginsenoside Rb1 improved lipid accumulation. Thus, ginsenoside Rb1 treatment significantly improved inflammation, leptin resistance, and lipid accumulation in diabetic mice.

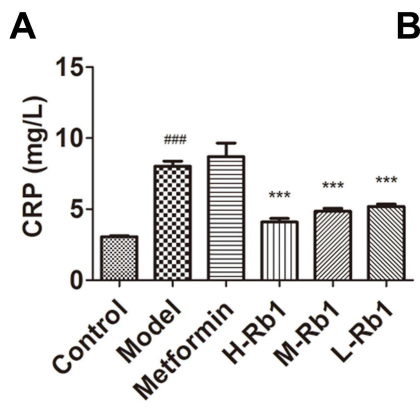

E

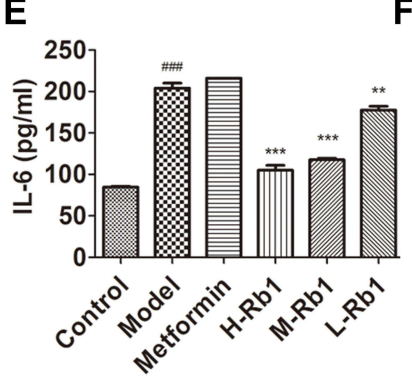

I
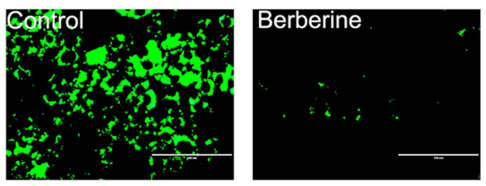

B
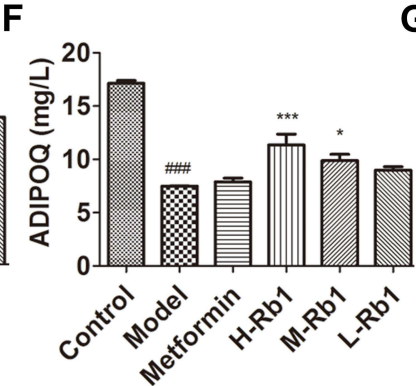

C

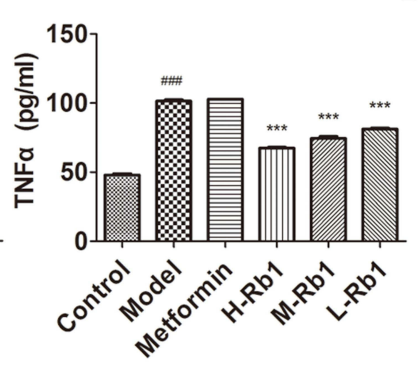

H

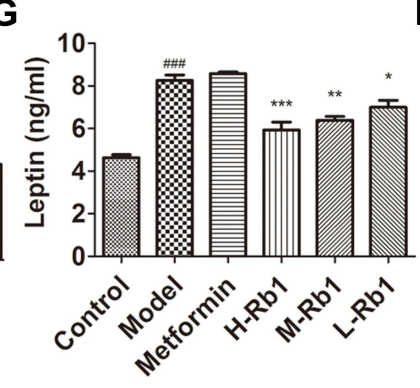

D
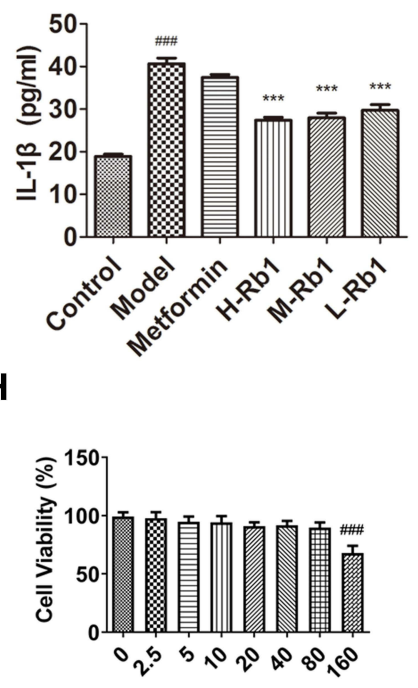

Ginsenoside Rb1 concentration $(\mu \mathrm{M})$

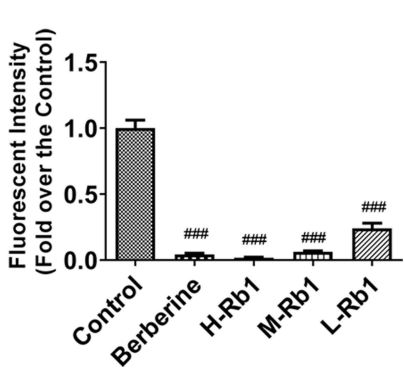

Figure 2 Ginsenoside RbI improved secretion of adipocytokines and lipid accumulation in adipocytes. (A) CRPI; (B) MCPI; (C) TNF $\alpha$; (D) IL-I $\beta$; (E) IL-6; (F) adiponectin levels and $(\mathbf{G})$ leptin in sera of different groups. (H) MTT assay showing 3T3-LI cell viability treated using different concentrations of ginsenoside RbI. (I) BODIPY staining

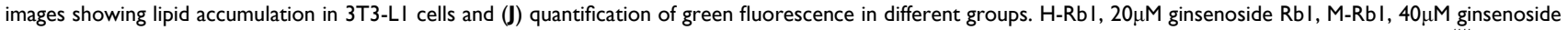

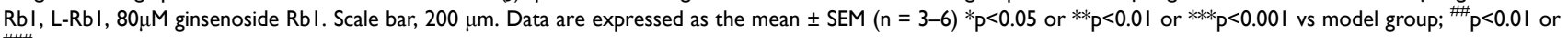
\#\# $\mathrm{p}<0.00$ I vs the control. 


\section{Ginsenoside Rbl Alleviated Fat Accumulation in the Liver}

The livers of the model mice exhibited increased lipid accumulation compared to those of the control group (Figure S1A and B, p $<0.001$ ), while treatment with metformin and ginsenoside $\mathrm{Rb} 1$ notably reduced lipid droplet levels in the livers $(\mathrm{p}<0.001)$. HE staining results revealed enlarged and vacuolar liver cells and inflammation of inflammatory cells in the model mice. However, treatment with both metformin and ginsenoside Rb1 significantly improved the cellular morphology and alleviated inflammation (Figure S1C). Moreover, the levels of ALT and AST, which reflect liver dysfunction, were significantly decreased in the $\mathrm{H}-\mathrm{Rb} 1$ treated $\mathrm{db} / \mathrm{db}$ mice (Figure S1D and E, p < 0.05), while M-Rb1 treated mice exhibited an improvement only in ALT levels. Conversely, neither L-Rb1 nor metformin treatment significantly improved ALT and AST levels ( $p>0.05)$.

\section{Ginsenoside RbI Improved Cardiac Function in Diabetic Mice}

Representative echocardiographic images of the six groups are shown in Figure 3A. Both LVAWd and LVPWd significantly decreased in the model mice but were improved by $\mathrm{H}-\mathrm{Rb} 1$ treatment (Figure $3 \mathrm{~B}$ and $\mathrm{C}, \mathrm{p}<0.05$ ). LVIDd was significantly increased in the model group compared to its levels in the control group (Figure 3D, $p<0.001$ ). In contrast, LVIDd significantly decreased in the H-Rb1 group compared with the model group $(\mathrm{p}<0.05)$. These data indicated amelioration of dilated cardiomyopathy in ginsenoside Rb1-treated diabetic mice. EF and FS were significantly lower in the model mice than in the control

A
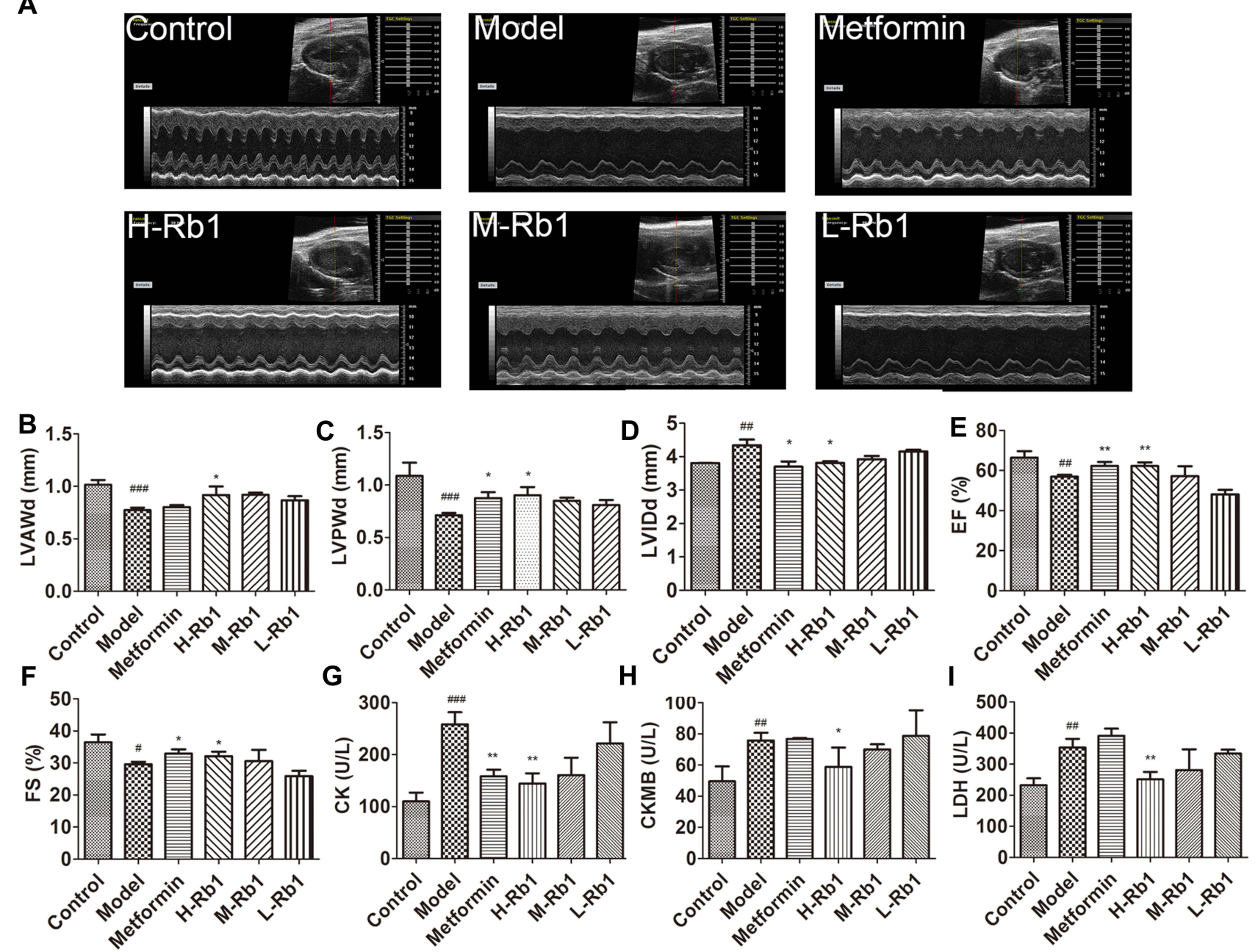

Figure 3 Ginsenoside RbI ameliorated cardiac dysfunction in diabetic mice. (A) Representative images of echocardiography from mice after 12 weeks of treatment. Statistical analyses of (B) LVAWd; (C) LVPWd; (D) LVIDd; (E) EF and (F) FS in indicated groups. Serum (G) CK; (H) CK-MB and (I) LDH in indicated groups. Data are expressed as the mean \pm SEM $(n=3-6)$. ${ }^{*} p<0.05$ or ${ }^{*}{ }^{p}<<0.01$ vs model group; ${ }^{\#}<0.05$ or ${ }^{\# \#} p<0.01$ or ${ }^{\# \#} \mathrm{p}<0.001$ vs the control. 
group (Figure $3 \mathrm{E}$ and $\mathrm{F}, \mathrm{p}<0.05$ ), indicating decreased systolic dysfunction and heart failure in the diabetic mice. However, $\mathrm{H}-\mathrm{Rb} 1$ administration significantly improved these parameters $(p<0.05)$, demonstrating that $H-R b 1$ can improve cardiac function in diabetic cardiomyopathy. Metformin administration significantly improved LVPWd, LVIDd, EF, and FS $(\mathrm{p}<0.05)$ but not LVAWd in the model group $(p>0.05)$. Elevated serum CK, CK-MB, and $\mathrm{LDH}$ levels reflected cardiac dysfunction in the model group; however, their levels were lower in the $\mathrm{H}-\mathrm{Rb} 1$ group (Figure 3G-I, $\mathrm{p}<0.05$ ), indicating that $\mathrm{H}-\mathrm{Rb} 1$ treatment improved cardiac function in diabetic mice. Metformin, however, improved only CK levels, and both $\mathrm{M}-\mathrm{Rb} 1$ and $\mathrm{L}-\mathrm{Rb} 1$ treatments failed to improve CK, CK-MB, and LDH levels.

\section{Ginsenoside RbI Attenuated Hypertrophy, Inflammation, Fibrosis, and Apoptosis in Diabetic Hearts}

HE and Masson's staining showed increased cross-sectional area and interstitial fibrosis of atrial cardiomyocytes in the model mice compared to the control mice
(Figure 4A-D, p $<0.001$ ); these increases were attenuated by ginsenoside $\mathrm{Rb} 1$ and metformin treatments $(\mathrm{p}<0.05)$. However, despite a decrease in cross-sectional area, there were no significant differences observed in the L-Rb1 group ( $p>0.05)$. Increased inflammatory cell infiltration and mineralization were observed in the diabetic hearts; however, both ginsenoside $\mathrm{Rb} 1$ and metformin administration improved cardiac morphology. TUNEL assay results showed significant increase in apoptosis in the model group (Figure 4E and F, p $<0.001$ ), while ginsenoside $\mathrm{Rb} 1$ and metformin treatment decreased the apoptotic rate in diabetic hearts $(\mathrm{p}<0.001)$. These data demonstrated that the administration of ginsenoside Rb1 alleviated cardiac dysfunction in diabetic mice by reducing hypertrophy, fibrosis, and apoptosis.

\section{Ginsenoside RbI Promoted Adipocytokine Signaling, as Well as Lipid and Glucose Metabolism, in Diabetic Hearts}

To explore the effects of ginsenoside Rb1 on lipid droplet formation, Oil Red $\mathrm{O}$ staining was performed to quantify

\section{A}

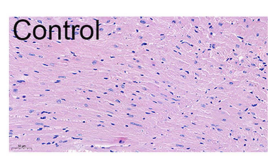

$\mathrm{H}-\mathrm{Rb} 1$

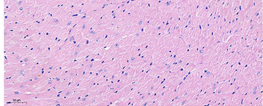

C
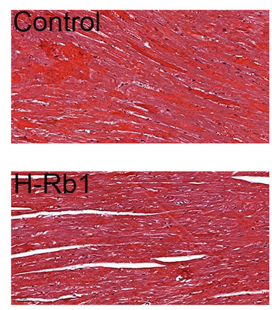

E
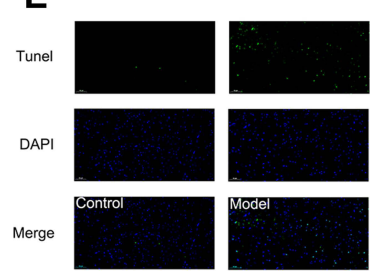
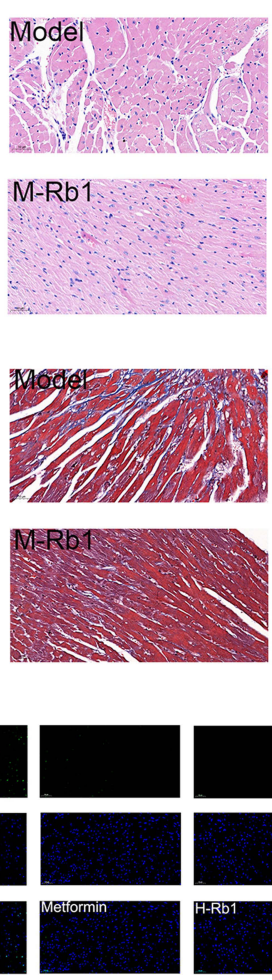
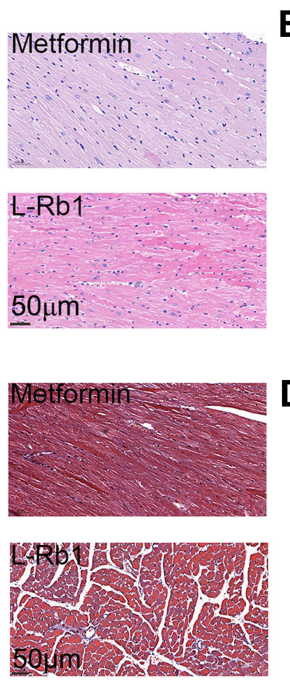

D
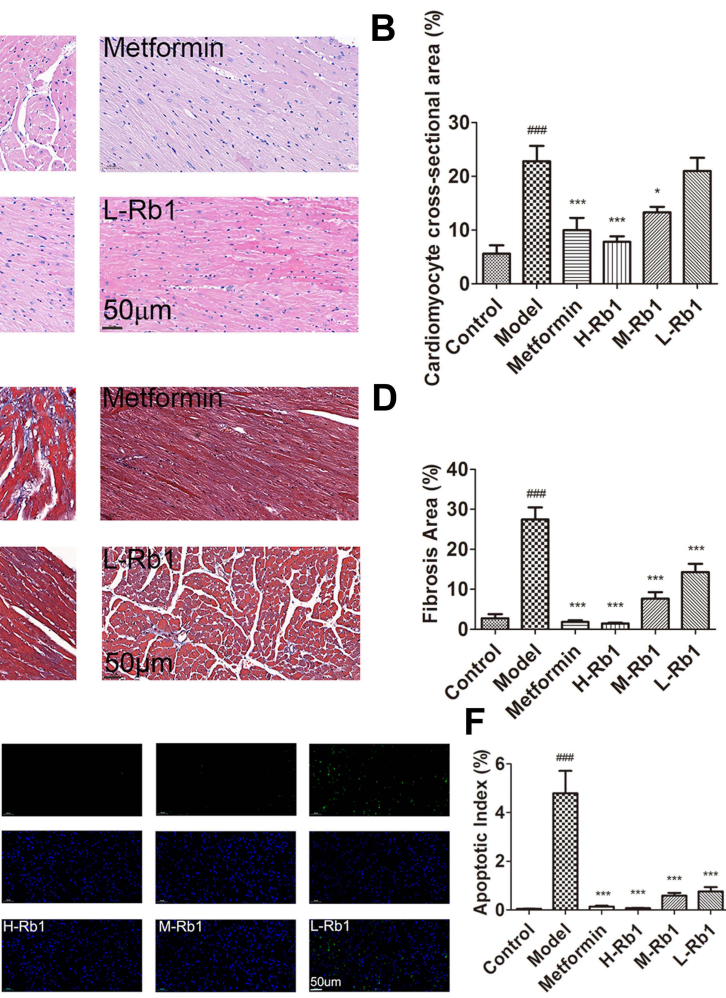

Figure 4 Ginsenoside Rbl reduced myocardial inflammation, hypertrophy, fibrosis, and apoptosis in diabetic mice. (A) Representative images of hematoxylin-eosin (HE) staining and (B) cross-sectional areas in indicated groups. (C) Representative images of Masson staining and (D) statistical analyses of fibrotic ratios in different groups. (E) Representative TUNEL staining images and (F) statistical analyses of cardiomyocyte apoptotic index in six groups. Scale bar, $50 \mu \mathrm{m}$. Data are expressed as the mean \pm SEM ( $\mathrm{n}$ $=3-6) .{ }^{* * *} p<0.001$ or ${ }^{*} p<0.05$ vs model group; ${ }^{\# \#} p<0.001$ vs the control. 
lipid accumulation in the cardiomyocytes (Figure 5A). A large number of lipid droplets were observed in the hearts of $\mathrm{db} / \mathrm{db}$ mice. The administration of ginsenoside $\mathrm{Rb} 1$ and metformin decreased lipid accumulation the heart (Figure 5B, p < 0.001). H9c2 cells were treated with PA to induce lipotoxicity, and the MTT assay was used to establish the suitable concentrations of PA and ginsenoside $\mathrm{Rb} 1$. Cell viability reduced to $60 \%$ after incubation with $200 \mu \mathrm{M} \mathrm{PA}$, and significantly increased after treatment with ginsenoside Rb1 (Figure S2A-C, $\mathrm{p}<0.01$ ). Therefore, $200 \mu \mathrm{M}$ PA-treated cells were used as the model group, and the H9c2 cells were pre-incubated with ginsenoside Rb1 before adding PA. BODIPY staining in H9c2 cells showed few small lipid droplets, whereas incubation with PA increased the number of droplets (Figure S2D and E, $\mathrm{p}<0.001$ ). However, the additions of ginsenoside $\mathrm{Rb} 1$ and berberine significantly decreased the number of lipid droplets compared with that of the model group, as evidenced by a decrease in green fluorescence in the ginsenoside $\mathrm{Rb} 1$ - and berberine-treated $\mathrm{H} 9 \mathrm{c} 2$ cells. Overall, these data indicate that ginsenoside Rb1 administration reduced the accumulation of cytosolic lipid droplets.

To further elucidate the mechanisms underlying lipidlowering effects of ginsenoside $\mathrm{Rb} 1, \mathrm{H}-\mathrm{Rb} 1$ hearts were analyzed by qRT-PCR, and $80 \mu \mathrm{M}$ ginsenoside Rb1-treated $\mathrm{H} 9 \mathrm{c} 2$ cells were subjected to Western blotting. Expression of genes involved in the adipocytokine signaling pathway was detected. Treatment with ginsenoside $\mathrm{Rb} 1$ and metformin significantly improved the levels of insulin receptor substrate 1 ( $I r S 1$ ), adenosine 5'-monophosphate (AMP)-activated protein kinase 1 (Ampk1), Ampk2, adiponectin receptor 1 (Adipor1), and Adipor2, suggesting improved adipocytokine signaling (Figure $5 \mathrm{C}, \mathrm{p}<0.01$ ). Additionally, there was a significant decrease in the expression of genes involved in lipid metabolism, including peroxisome proliferative activated receptor $\alpha$ (Ppar $\alpha$ ), Ppar $\gamma$ coactivator $1 \alpha(P g c 1 \alpha)$, malonyl-CoA decarboxylase $(M l y c d)$, acyl-CoA dehydrogenase (Acadm), acylcoenzyme A oxidase 1 (Acox1), acetyl-COA acyltransferase 2 (Acaa2), acetyl CoA carboxylase B (Acacb), acetyl coenzyme A synthetase 2 (Acss2), stearoyl-CoA

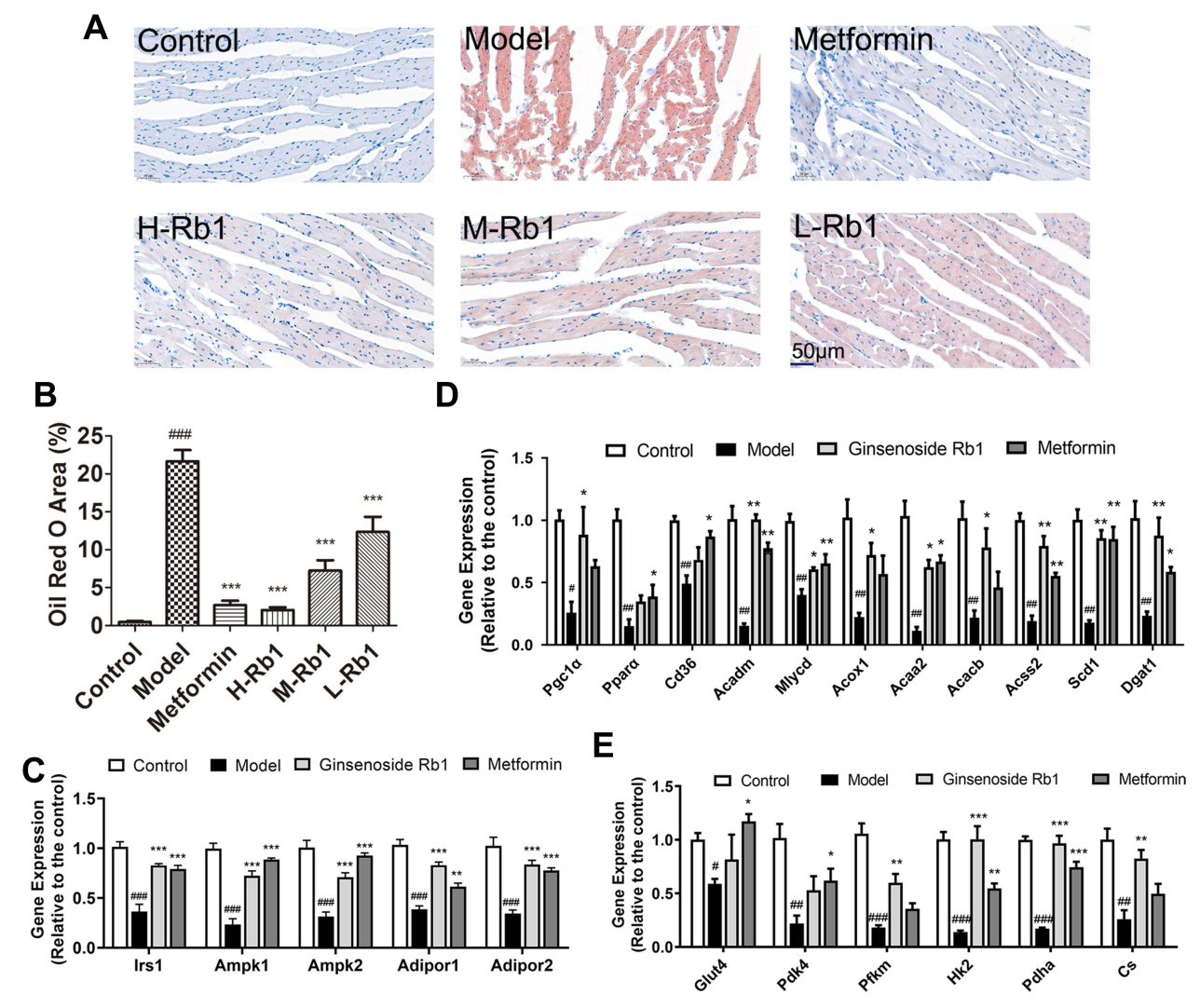

Figure 5 Ginsenoside RbI reduced lipid accumulation and promoted adipocytokine-mediated glucose and lipid metabolism in diabetic heart. (A) Representative images of Oil red $\mathrm{O}$ staining and (B) quantitative analysis of Oil red $\mathrm{O}$ staining in six groups. Scale bar, $50 \mu \mathrm{m}$. Transcription levels of genes involved in (C) adipocytokine pathway, (D) lipid and (E) glucose metabolism in indicated four groups. Data are expressed as the mean \pm SEM $(n=3-6) .{ }^{*} p<0.05$ or $* * p<0.01$ or $* * * p<0.00$ I vs. model group; ${ }^{*} \mathrm{p}<0.00$ I or ${ }^{\#}<0.01$ or ${ }^{\#} \mathrm{p}<0.05$ vs the control. 
desaturase $1(S c d 1)$, and diacylglycerol acyltransferase 1 (Dgat1), in the diabetic hearts after 36 weeks (Figure 5D, $\mathrm{p}<0.05)$. Furthermore, the expression of $\mathrm{Cd} 36$, which plays a critical role in lipid transport, was decreased in the model group $(\mathrm{p}<0.05)$. Treatment with $\mathrm{H}-\mathrm{Rb} 1$ significantly increased their expression $(p<0.05)$, indicating that $\mathrm{H}-\mathrm{Rb} 1$ treatment improved lipid metabolism in diabetic hearts. Treatment with metformin, however, only partially improved the expression of these proteins. Accordingly, in $\mathrm{H} 9 \mathrm{c} 2$ cells, the protein levels of PGC1 $\alpha, \operatorname{PPAR} \alpha, \mathrm{CD} 36$, carnitine palmitoyltransferase 1a (CPT1A), CPT1B, and CPT2 were significantly decreased in the model group compared with those in the control group (Figure S2F and $\mathrm{G}, \mathrm{p}<0.05)$. Ginsenoside Rb1 and berberine treatment significantly improved their levels $(\mathrm{p}<0.05)$, which reduced lipid accumulation in cardiomyocytes and provided sufficient energy for normal cardiac function. The expression of genes related to glucose metabolism, such as glucose transporter 4 (Glut4), pyruvate dehydrogenase kinase $4(P d k 4)$, phosphofructokinase $(P f k m)$, hexokinase 2 (Hk2), pyruvate dehydrogenase $\alpha(P d h a)$, and citric acid synthase $(C s)$, was significantly decreased in the diabetic hearts (Figure 5E, p < 0.05). Treatment with ginsenoside Rb1 significantly improved the expression of Pfkm, Hk2, $P d h a$, and $C s(\mathrm{p}<0.05)$. Although treatment with ginsenoside Rb1 increased the protein levels of GLUT4 and PDK4 in $\mathrm{H} 9 \mathrm{c} 2$ cells $(\mathrm{p}<0.05)$, the respective gene expression level did not improve in the diabetic hearts. These data indicated that ginsenoside $\mathrm{Rb} 1$ reduced lipid accumulation in diabetic hearts by promoting lipid metabolism, and accelerating glucose metabolism to alleviate insulin resistance.

\section{Ginsenoside RbI Reduced ROS Accumulation in Mitochondria}

Serum levels of MDA, SOD, GSH-Px, and CAT proteins were quantified, and the diabetic mice showed reduced antioxidative function. The levels of these antioxidant enzymes increased after treatment with ginsenoside $\mathrm{Rb} 1$ (Figure S3A-D, p < 0.01), whereas treatment with metformin did not significantly improve the antioxidative function of diabetic mice $(p>0.05)$. H9c2 cells exposed to PA showed significant increase in mitochondrial superoxide levels, as evidenced by increased fluorescence intensity. In contrast, pretreatment with berberine or ginsenoside $\mathrm{Rb} 1$ reduced mitochondrial superoxide levels (Figure S3E and F, $\mathrm{p}<0.05)$. Concomitantly, the gene expression of NAD
(P)H:quinone oxidoreductase 1 (Nqol), nuclear respiratory factor 2 (Nrf2), glutathione S-transferase (Gst), Sod1, Kelch-like ECH-associated protein 1 (Keap1), glutamate cysteine ligase catalytic subunit $(G c l c)$, and glutamate cysteine ligase modifier subunit $(\mathrm{Gclm})$ was significantly reduced in the model group compared to that in the control (Figure S3G, $\mathrm{p}<0.05$ ). Treatment with ginsenoside Rb1 or berberine significantly increased the expression of these genes $(\mathrm{p}<0.05)$, suggesting that ginsenoside $\mathrm{Rb} 1$ improved the PA-suppressed antioxidative function of the cardiomyocytes. Collectively, our results provide evidence that ginsenoside $\mathrm{Rb} 1$ treatment reduced ROS accumulation and increased antioxidant function.

\section{Discussion}

The principal approach to control diabetes is lowering body weight and blood glucose levels. In this study, treatment with ginsenoside $\mathrm{Rbl}$ prevented weight gain and reduced blood glucose and lipid levels in diabetic mice, as reported previously. ${ }^{18}$ Importantly, our study clarifies the mechanism of ginsenoside Rb1 in influencing adipocytokine pathways and protecting against diabetic cardiomyopathy. Treatment with ginsenoside $\mathrm{Rb} 1$ improved levels of serum IL- $1 \beta$, TNF- $\alpha$, MCP-1, IL-6, and CRP, as well as decreased leptin and adiponectin levels. Lipid accumulation and inflammation in the adipocytes, liver, and heart were also alleviated; as a result, improved cardiac function, as well as reduced oxidative stress, hypertrophy, fibrosis, and apoptosis were observed in the heart. This study has demonstrated that ginsenoside Rb1 regulates the adipocytokine pathway, which mediates glucose and lipid metabolism, thereby activating anti-oxidative pathways and protecting against diabetic cardiomyopathy (Figure 6).

Treatment with ginsenoside Rb1 significantly improved serum lipid levels in diabetic mice; this result may be attributed to the improvement in adipose function. Adipose tissue is regarded as a sink for excess energy storage in the form of triglycerides, and a reservoir from which FFAs are released. ${ }^{19}$ Obesity-related inflammation is characterized by increased levels of serum cytokines, including CRP, MCP-1, IL-1 $\beta$, IL-6, and TNF- $\alpha$. Adipose dysfunction can lead to an increased release of TGs and FFAs from adipose tissues, elevating serum TG and FFA levels. ${ }^{20}$ In response to elevated serum lipid levels, the levels of HDL and LDL, which serve as carriers in lipid transport, also increase, leading to elevated serum $\mathrm{CHO}$ levels. When treated with ginsenoside Rb1, 3T3-L1 


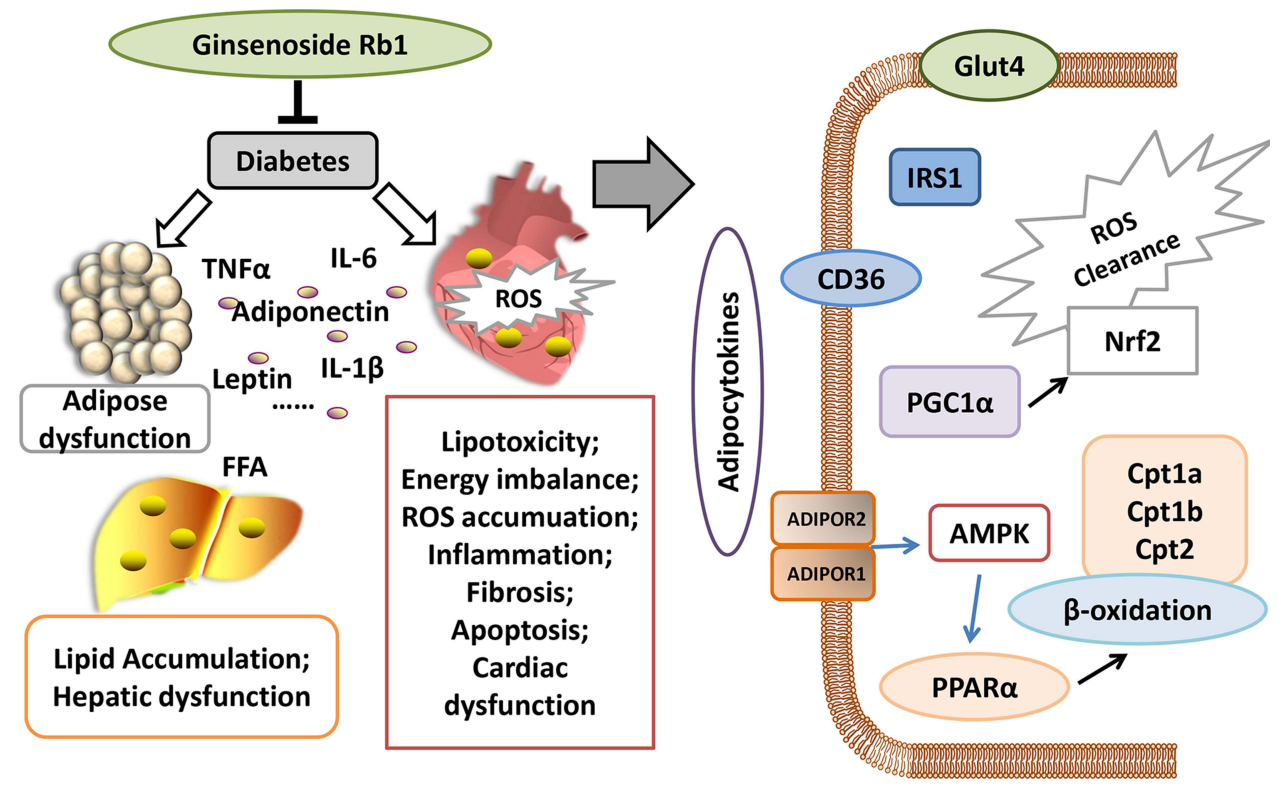

Figure 6 Schematic figure illustrating the mechanism of ginsenoside RbI in treating diabetic cardiomyopathy. Under obese or diabetic conditions, adipose function is impaired, and excess lipids and abnormal adipocytokines are released into the serum, thereby influencing systemic organs, including the heart. In diabetic heart, more fatty acids are utilized to generate energy, producing excessive ROS that accumulate in cardiomyocytes and induce oxidative stress. Oxidative stress and pro-inflammatory cytokines from the adipose tissue can cause cardiac inflammation, fibrosis, and apoptosis, subsequently leading to cardiac dysfunction and heart failure. The present study showed that ginsenoside Rbl improved adipocytokine pathway, improved the body weight as well as body fat and serum lipids of diabetic mice; these changes influenced cardiac glucose and lipid metabolism and exerted a preventive effect on diabetic cardiomyopathy.

preadipocytes in the diabetic mice displayed reduced number of lipid droplets. Excessive accumulation of hepatic lipids was observed due to increased release of FFAs from the adipose tissues. Increased lipid accumulation in liver plays an important role in the induction of hepatic insulin resistance and impaired hepatic function. ${ }^{3}$ Furthermore, elevated levels of ALT and AST, which indicate hepatic dysfunction, were observed in the diabetic mice. However, ginsenoside $\mathrm{Rb} 1$ administration improved adipose tissue function, serum lipid levels, and hepatic lipid accumulation.

Previous studies have indicated that diabetes can lead to thinning of ventricular wall, dysfunction of myocardial contraction, and enlarged left ventricular volume. ${ }^{21}$ Consistently, we demonstrated significant changes in the histopathology and function of the diabetic heart compared with control specimens. ${ }^{22}$ HE staining revealed disorganized fibers, nuclear loss, and infiltration of inflammatory cells in the hearts of diabetic mice. In addition, fibrosis and apoptosis of cardiomyocytes were observed; however, these factors were alleviated following treatment with ginsenoside Rb1. Diabetic mice also exhibited high levels of serum CK, CK-MB, and LDH, which indicated significant myocardial damage. ${ }^{23}$ However, treatment with ginsenoside $\mathrm{Rb} 1$ for 12 weeks significantly alleviated signs of this damage. As expected, cardiac function parameters such as LVAWd, LVPWd, EF, and FS decreased in the model group, indicating the thinning of the ventricular wall and myocardial contractile dysfunction. Cardiac dysfunction was also indicated following an enlarged left ventricular volume due to the significant increase of LVIDd in diabetic mice. However, treatment with ginsenoside $\mathrm{Rb} 1$ for 12 weeks significantly increased LVAWd, LVPWd, EF, and FS, and decreased LVIDd. These data suggest that ginsenoside Rb1 can significantly improve cardiac dysfunction and myocardial injury induced by diabetes.

Early-stage diabetic cardiomyopathy is characterized by intracellular lipid accumulation, hypertrophy, inflammation, fibrosis, and diastolic dysfunction, which eventually develops into systolic dysfunction and heart failure. ${ }^{24}$ Abnormal myocardial lipid metabolism may lead to excessive ROS generation, which is an important cause of inflammation, hypertrophy, and fibrosis of cardiomyocytes in diabetic mice. ${ }^{25,26}$ In this study, diabetic mice showed a significant increase in circulating lipids and pro-inflammatory cytokine levels compared to control mice, as well as decreased levels of antioxidative markers that reportedly play crucial roles in the development of myocardial hypertrophy and dysfunction. ${ }^{27}$ However, 
diabetic mice treated with ginsenoside Rb1 showed a significant reduction in serum lipids and inflammatory cytokine levels, along with improved antioxidative function. Furthermore, ginsenoside Rbl improved myocardial fibrosis, as evidenced by a decreased collagen area following Masson's staining. ROS accumulation and myocardial inflammation also induced myocardial apoptosis, which was markedly reduced following treatment with ginsenoside Rb1 (Figure 4).

Mechanistic investigation revealed that the cardio-protective effect of ginsenoside $\mathrm{Rb} 1$ was mediated by the adipocytokine-pathway. Adipose tissue exerts direct effects on systemic homeostasis by secreting a wide range of bioactive adipocytokines, including CRP, MCP-1, IL-1 $\beta$, IL-6, TNF- $\alpha$, adiponectin, and leptin. ${ }^{28}$ Ginsenoside Rb1 treatment reduced the secretion of pro-inflammatory cytokines such as IL- 6 and TNF- $\alpha$, which induce insulin resistance by inhibiting GLUT4 expression, thereby reducing the phosphorylation of IRS1. ${ }^{29}$ Obesity is often accompanied by chronic inflammation, and inflammation of organs such as the liver, adipose tissue, or heart impacts energy homeostasis, influencing organic function. ${ }^{30}$ IL-6 may influence expression of Glut4, Irs 1, and Ampk, which regulate lipid and glucose metabolism. ${ }^{31}$ TNF- $\alpha$ can also downregulate the expression of PGC1 $\alpha, \mathrm{PDK} 4$, and CPT1, which play critical roles in regulating energy metabolism. ${ }^{32}$ Elevated levels of circulating pro-inflammatory cytokines also indicate poor prognosis in patients with heart failure because activation of immune response in the heart leads to adverse cardiac remodeling and left ventricular dysfunction. ${ }^{33}$ In this study, ginsenoside $\mathrm{Rb} 1$ reduced the levels of circulating CRP, MCP-1, IL-1 $\beta$, IL-6 and TNF- $\alpha$, indicating alleviation of global inflammation and improved energy homeostasis.

Increased serum adiponectin activates the AMPK$\operatorname{PPAR} \alpha$ pathway in cardiomyocytes, and enhances glucose and lipid metabolism to promote energy homeostasis. Activated fatty acid $\beta$-oxidation also reduces lipid accumulation in the myocardium. ${ }^{34}$ Further analyses showed that the increased adiponectin directly bound to ADIPOR1 and ADIPOR2, activated the PPAR $\alpha$ pathway, and promoted glucose and lipid metabolism by increasing GLUT4, PDK4, and CD36 expression to achieve energy homeostasis. Fatty acid oxidation was also promoted, as evidenced by elevated levels of CPT1A, CPT1B, and CPT2, which reduced lipid accumulation in cardiomyocytes and alleviated inflammation and oxidative stress caused by excessive lipid droplets. Hyperlipidemia and insulin resistance in diabetes result in lower glucose utilization and conversion of excessive fatty acids into TGs for storage in the cardiomyocytes. ${ }^{35}$ Our study demonstrated that the number of lipid droplets decreased in the ginsenoside Rb1-treated diabetic hearts due to its lipid-lowering effect, which was confirmed in the PAtreated $\mathrm{H} 9 \mathrm{c} 2$ cardiomyocytes.

Activation of $\mathrm{PGC} 1 \alpha-\mathrm{Nrf} 2$, the downstream pathway of leptin promoted antioxidative function in cardiomyocytes. The expression and secretion of leptin are closely associated with body fat and insulin levels. ${ }^{36}$ Leptin can affect the contractility of cardiomyocytes and contribute to altered myocardial function. ${ }^{37}$ Therefore, leptin concentration may influence various cardiovascular diseases, including acute myocardial infarction, stroke, coronary heart disease, chronic heart failure, and left cardiac hypertrophy. ${ }^{38}$ In $\mathrm{db} / \mathrm{db}$ mice, leptin signaling was inhibited owing to a lack of leptin receptors; thus, the protective effects of leptin on the heart were impaired. However, excessive serum leptin levels were reduced by ginsenoside $\mathrm{Rb} 1$, and the activated PGC1- $\alpha$ pathway neutralized some of the effects exerted by the loss of leptin receptors. Through the PGC1 $\alpha$-Nrf2 pathway, treatment with ginsenoside Rb1 improved expression of Keap1, Gclc, Gclm, and Sod, which exerted antioxidative effects on the diabetic heart. Accordingly, decreased ROS accumulation was observed in the ginsenoside Rb1-treated cardiomyocytes, indicating alleviation of oxidative stress. Therefore, both glucose and lipid metabolism, through antioxidative pathways regulated by adipocytokines, mediate the preventive effect of ginsenoside $\mathrm{Rb} 1$ on diabetic cardiomyopathy.

Despite these conclusions, this study has some limitations. First, we did not determine whether ginsenoside Rb1 improved diabetic cardiomyopathy by directly targeting cardiomyocytes, or through an indirect lipid lowering effect. Second, how ginsenoside Rb1 mediated regulation of the adipocytokine pathway and its specific target remained to be completely elucidated. Finally, we only focused on lipotoxicity for initiation and development of diabetic cardiomyopathy, and the effect of ginsenoside $\mathrm{Rb} 1$ on hyperglycemia and insulin resistance related pathways remains to be further investigated.

\section{Conclusion}

Ginsenoside Rb1 improved the body weight, body fat, and adipocytokine levels in diabetic mice, thus regulating adipose function in these animals. Through an adipocytokinemediated pathway, ginsenoside $\mathrm{Rbl}$ modulated glucose 
and lipid metabolism and attenuated hyperglycemia/hyperlipidemia-induced oxidative stress in the myocardium of diabetic mice. Additionally, ginsenoside Rb1 exerted strong inhibitory effects against cardiac hypertrophy, inflammation, fibrosis, and apoptosis caused by excess lipids and ROS. Therefore, ginsenoside Rb1 may be a useful drug for preventing diabetic cardiomyopathy that acts via modulating the adipocytokine pathway, improving lipid and glucose metabolism, reducing inflammation, and promoting antioxidative function.

\section{Abbreviations}

$\mathrm{H}-\mathrm{Rb} 1$, ginsenoside Rb1 $100 \mathrm{mg} / \mathrm{kg} /$ day; M-Rb1, ginsenoside Rb1 50 mg/kg/day; L-Rb1, ginsenoside Rb1 25 $\mathrm{mg} / \mathrm{kg} /$ day; PA, palmitate acid; TG, total triglyceride; $\mathrm{CHO}$, total cholesterol; HDL, high-density lipoprotein; LDL, low-density lipoprotein; FFA, free fatty acid; ALT, alanine aminotransferase; AST, aspartate aminotransferase; CK, creatine kinase; LDH, lactate dehydrogenase; CAT, catalase; MDA, malondialdehyde; SOD, superoxide dismutase; GSH-px, glutathione peroxidase; IL-6, interleukin- 6; MCP-1, monocyte chemoattractant protein-1; TNF- $\alpha$, tumor necrosis factor- $\alpha$; CRP, C-reactive protein; LVID, left ventricular internal diameter; EF, left ventricular ejection fraction; FS, fractional shortening; LVAW, left ventricular anterior wall thickness; PGC1 $\alpha$, PPAR $\gamma$ coactivator $1 \alpha$; IRS1, insulin receptor substrate 1; AMPK, adenosine 5'-monophosphate (AMP)-activated protein kinase; ADIPOR, adiponectin receptor; MLYCD, malonyl-CoA decarboxylase; ACADM, acyl-CoA dehydrogenase; ACOX1, acyl-coenzyme A oxidase 1; ACAA2, acetyl-COA acyltransferase 2; ACACB, acetyl CoA carboxylase B; ACSS2, acetyl coenzyme A synthetase 2; SCD1, stearoyl-CoA desaturase 1; DGAT1, diacylglycerol acyltransferase 1; CPT1A, carnitine palmitoyltransferase 1a; GLUT4, glucose transporter 4; PDK4, pyruvate dehydrogenase kinase 4; PFKM, phosphofructokinase; HK2, hexokinase 2; PDHA, pyruvate dehydrogenase $\alpha$; CS, citric acid synthase; NQO1, NAD(P)H:quinone oxidoreductase 1; NRF2, nuclear respiratory factor 2; GST, glutathione S-transferase; KEAP1, Kelch-like ECH-associated protein 1; GCLC, glutamate cysteine ligase catalytic subunit; GCLM, glutamate cysteine ligase modifier subunit.

\section{Data Sharing Statement}

All data and materials are available upon request.

\section{Ethics Approval}

The animal experiments were approved by the Laboratory Animal Ethics Committee of the Institute of Medicinal Plant Development, Peking Union Medical College (SLXD-20200113001).

\section{Consent for Publication}

All authors have declared their consent for this publication.

\section{Author Contributions}

All authors have read and agreed to the published version of the manuscript. All authors made a significant contribution to the work reported, whether that is in the conception, study design, execution, acquisition of data, analysis and interpretation, or in all these areas; took part in drafting, revising or critically reviewing the article; gave final approval of the version to be published; have agreed on the journal to which the article has been submitted; and agree to be accountable for all aspects of the work.

\section{Funding}

This study was supported by National Natural Science Foundation of China (Grant No.81773936), and Science and technology planning project of Yunnan Science and Technology Department (Grant No.202102AA310048).

\section{Disclosure}

The authors report no conflicts of interest in this work.

\section{References}

1. Salas-Salvado J, Martinez-Gonzalez MA, Bullo M, Ros E. The role of diet in the prevention of type 2 diabetes. Nutr Metab Cardiovasc Dis. 2011;21(Suppl 2):B32-B48. doi:10.1016/j.numecd.2011.03.009

2. Reaven GM. Banting lecture 1988. Role of insulin resistance in human disease. Diabetes. 1988;37(12):1595-1607. doi:10.2337/ diab.37.12.1595

3. Girard J, Lafontan M. Impact of visceral adipose tissue on liver metabolism and insulin resistance. Part II: visceral adipose tissue production and liver metabolism. Diabetes Metab. 2008;34(5):439445. doi:10.1016/j.diabet.2008.04.002

4. Jaganathan R, Ravindran R, Dhanasekaran S. Emerging role of adipocytokines in type 2 diabetes as mediators of insulin resistance and cardiovascular disease. Can J Diabetes. 2018;42(4):446-456 e441. doi:10.1016/j.jcjd.2017.10.040

5. Oikonomou EK, Antoniades C. The role of adipose tissue in cardiovascular health and disease. Nat Rev Cardiol. 2019;16(2):83-99. doi:10.1038/s41569-018-0097-6

6. Jia G, Whaley-Connell A, Sowers JR. Diabetic cardiomyopathy: a hyperglycaemia- and insulin-resistance-induced heart disease. Diabetologia. 2018;61(1):21-28. doi:10.1007/s00125-017-4390-4 
7. Jia G, DeMarco VG, Sowers JR. Insulin resistance and hyperinsulinaemia in diabetic cardiomyopathy. Nat Rev Endocrinol. 2016;12 (3):144-153. doi:10.1038/nrendo.2015.216

8. Carpentier AC. Abnormal myocardial dietary fatty acid metabolism and diabetic cardiomyopathy. Can J Cardiol. 2018;34(5):605-614. doi:10.1016/j.cjca.2017.12.029

9. Zorena K, Jachimowicz-Duda O, Slezak D, Robakowska M, Mrugacz M. Adipokines and obesity. Potential link to metabolic disorders and chronic complications. Int J Mol Sci. 2020;21:10. doi:10.3390/ ijms 21103570

10. Zhao S, Kusminski CM, Scherer PE. Adiponectin, leptin and cardiovascular disorders. Circ Res. 2021;128(1):136-149. doi:10.1161/ CIRCRESAHA.120.314458

11. Cao H. Adipocytokines in obesity and metabolic disease. $J$ Endocrinol. 2014;220(2):T47-T59. doi:10.1530/JOE-13-0339

12. Zhou P, Xie W, He S, et al. Ginsenoside Rb1 as an anti-diabetic agent and its underlying mechanism analysis. Cells. 2019;8(3):204. doi: $10.3390 /$ cells 8030204

13. Lim S, Park J, Um JY. Ginsenoside Rb1 induces beta 3 adrenergic receptor-dependent lipolysis and thermogenesis in 3T3-L1 adipocytes and db/db mice. Front Pharmacol. 2019;10:1154. doi:10.3389/ fphar.2019.01154

14. Park SJ, Park M, Sharma A, Kim K, Lee HJ. Black ginseng and ginsenoside Rb1 promote browning by inducing UCP1 expression in 3T3-L1 and primary white adipocytes. Nutrients. 2019;11:11. doi:10.3390/nu11112747

15. Jiang L, Yin X, Chen YH, et al. Proteomic analysis reveals ginsenoside Rb1 attenuates myocardial ischemia/reperfusion injury through inhibiting ROS production from mitochondrial complex I. Theranostics. 2021;11(4):1703-1720. doi:10.7150/thno.43895

16. Qin L, Wang J, Zhao R, Zhang X, Mei Y. Ginsenoside-Rb1 improved diabetic cardiomyopathy through regulating calcium signaling by alleviating protein O-GlcNAcylation. J Agric Food Chem. 2019;67 (51):14074-14085. doi:10.1021/acs.jafc.9b05706

17. Zhu X, Yang J, Zhu W, et al. Combination of berberine with resveratrol improves the lipid-lowering efficacy. Int J Mol Sci. 2018;19 (12):3903. doi:10.3390/ijms19123903

18. Guo R, Wang L, Zeng $\mathrm{X}$, et al. Aquaporin 7 involved in GINSENOSIDE-RB1-mediated anti-obesity via peroxisome proliferator-activated receptor gamma pathway. Nutr Metab. 2020;17:69. doi:10.1186/s12986-020-00490-8

19. Kusminski CM, Bickel PE, Scherer PE. Targeting adipose tissue in the treatment of obesity-associated diabetes. Nat Rev Drug Discov. 2016;15(9):639-660. doi:10.1038/nrd.2016.75

20. Kawai T, Autieri MV, Scalia R. Adipose tissue inflammation and metabolic dysfunction in obesity. Am J Physiol Cell Physiol. 2021;320(3):C375-C391. doi:10.1152/ajpcell.00379.2020

21. Bugger H, Abel ED. Molecular mechanisms of diabetic cardiomyopathy. Diabetologia. 2014;57(4):660-671. doi:10.1007/s00125-0143171-6

22. Lorenzo-Almoros A, Tunon J, Orejas M, Cortes M, Egido J, Lorenzo O. Diagnostic approaches for diabetic cardiomyopathy. Cardiovasc Diabetol. 2017;16(1):28. doi:10.1186/s12933-017-0506-x
23. Al-Rasheed NM, Al-Rasheed NM, Hasan IH, et al. Simvastatin ameliorates diabetic cardiomyopathy by attenuating oxidative stress and inflammation in rats. Oxid Med Cell Longev. 2017;2017:1092015. doi:10.1155/2017/1092015

24. Dillmann WH. Diabetic cardiomyopathy. Circ Res. 2019;124 (8):1160-1162. doi:10.1161/CIRCRESAHA.118.314665

25. Zhou B, Tian R. Mitochondrial dysfunction in pathophysiology of heart failure. J Clin Invest. 2018;128(9):3716-3726. doi:10.1172/ JCI120849

26. Zhao XJ, Yu HW, Yang YZ, et al. Polydatin prevents fructoseinduced liver inflammation and lipid deposition through increasing miR-200a to regulate Keap1/Nrf2 pathway. Redox Biol. 2018;18:124-137. doi:10.1016/j.redox.2018.07.002

27. Schultheiss HP, Fairweather D, Caforio ALP, et al. Dilated cardiomyopathy. Nat Rev Dis Primers. 2019;5(1):32. doi:10.1038/s41572019-0084-1

28. Fasshauer M, Bluher M. Adipokines in health and disease. Trends Pharmacol Sci. 2015;36(7):461-470. doi:10.1016/j.tips.2015.04.014

29. Yaribeygi H, Farrokhi FR, Butler AE, Sahebkar A. Insulin resistance: review of the underlying molecular mechanisms. $J$ Cell Physiol. 2019;234(6):8152-8161. doi:10.1002/jcp.27603

30. Ravaut G, Legiot A, Bergeron K-F, Mounier C. Monounsaturated fatty acids in obesity-related inflammation. Int J Mol Sci. 2020;22 (1):330. doi:10.3390/ijms22010330

31. Marko DM, Foran G, Vlavcheski F, et al. Interleukin-6 treatment results in GLUT4 translocation and AMPK phosphorylation in neuronal SH-SY5Y cells. Cells. 2020;9(5):5. doi:10.3390/cells9051114

32. Gao F, Ni Y, Luo Z, et al. Atorvastatin attenuates TNF-alpha-induced increase of glucose oxidation through PGC-1alpha upregulation in cardiomyocytes. J Cardiovasc Pharmacol. 2012;59(6):500-506. doi:10.1097/FJC.0b013e31824c853c

33. Zhang Y, Bauersachs J, Langer HF. Immune mechanisms in heart failure. Eur J Heart Fail. 2017;19(11):1379-1389. doi:10.1002/ ejhf.942

34. Fang H, Judd RL. Adiponectin regulation and function. Compr Physiol. 2018;8(3):1031-1063.

35. Wang Z, Zhu Y, Zhang Y, et al. Protective effects of AS-IV on diabetic cardiomyopathy by improving myocardial lipid metabolism in rat models of T2DM. Biomed Pharmacother. 2020;127:110081. doi:10.1016/j.biopha.2020.110081

36. Angel Garcia-Merino J, Moreno-Perez D, de Lucas B, et al. Chronic flavanol-rich cocoa powder supplementation reduces body fat mass in endurance athletes by modifying the follistatin/myostatin ratio and leptin levels. Food Funct. 2020;11(4):3441-3450. doi:10.1039/ D0FO00246A

37. Nickola MW, Wold LE, Colligan PB, Wang GJ, Samson WK, Ren J. Leptin attenuates cardiac contraction in rat ventricular myocytes. Role of NO. Hypertension. 2000;36(4):501-505. doi:10.1161/01. HYP.36.4.501

38. Katsiki N, Mikhailidis DP, Banach M. Leptin, cardiovascular diseases and type 2 diabetes mellitus. Acta Pharmacol Sin. 2018;39(7):11761188. doi:10.1038/aps.2018.40
Journal of Inflammation Research

\section{Publish your work in this journal}

The Journal of Inflammation Research is an international, peerreviewed open-access journal that welcomes laboratory and clinica findings on the molecular basis, cell biology and pharmacology of inflammation including original research, reviews, symposium reports, hypothesis formation and commentaries on: acute/chronic inflammation; mediators of inflammation; cellular processes; molecular mechanisms; pharmacology and novel anti-inflammatory drugs; clinical conditions involving inflammation. The manuscript management system is completely online and includes a very quick and fair peerreview system. Visit http://www.dovepress.com/testimonials.php to read real quotes from published authors. 